\title{
Workforce Transitions and Social Connectedness Among Older Adults in the United States
}

Jason Settels, Markus H Schafer

Abstract:

As the industrialized world faces a rapidly aging population, it has become increasingly important to understand the factors that influence the well-being of older persons. In this regard, many scholars have emphasized the importance of social connectedness. Various theories seek to explain social connectedness in later life, particularly as it applies to workforce involvement. Among those theories, we engage the theories of activity-substitution and of complementarity, and we seek to discover which provides a better account of the social and work lives of older Americans. We do so through an analysis of the first 2 waves (Wave 1: 2005-2006, Wave 2: 2010-2011) of the National Social Life, Health, and Aging Project (NSHAP). Workforce transitions over time are assessed for their impact on 3 social connectedness outcomes: expansiveness of close networks, overall friendship network, and social participation. Results provide strong support for the theory of activity-substitution. Furthermore, our results show that those re-entering paid work after some time out of the workforce show the largest changes in social connectedness. The present study reinforces the importance of studying social connectedness as a multidimensional concept and draws attention to the increasing variability in workforce participation patterns among older persons.

https://doi.org/10.1093/workar/wax029

https://academic.oup.com/workar/article-abstract/4/3/274/4827939 